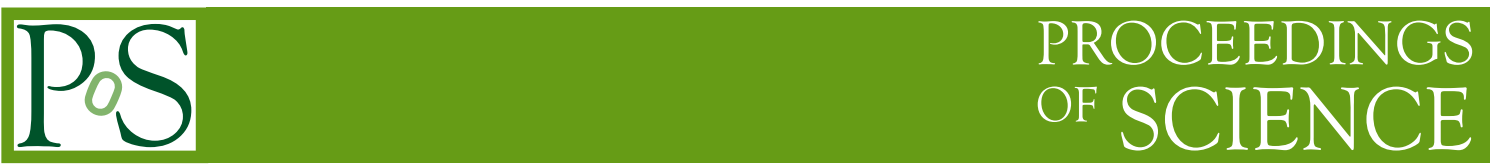

\title{
Oscillation Parameters Present: Session Summary
}

\author{
Inés Gil-Botella \\ Basic Research Department, High Energy Physics Division, CIEMAT, Madrid \\ E-mail: ines.gileciemat.es \\ Yoshi Uchida \\ Department of Physics, Imperial College London \\ E-mail: Yoshi.Uchida@imperial.ac.uk
}

\begin{abstract}
Session I of the Neutrino Oscillation Workshop 2018 Conference, "Neutrino Oscillations: Present", is summarised. Results were presented by the currently-running long-baseline oscillation experiments $\mathrm{T} 2 \mathrm{~K}$ and NOvA, as well as from the accelerator experiments OPERA and MiniBooNE. Status reports and results from experiments using short-baseline accelerator neutrinos (ICARUS and MicroBooNE), atmospheric neutrinos (Super-K, IceCube and ANTARES), and those from reactors (Daya Bay and Double Chooz), and from the Sun and the Earth (Borexino) were also presented. Our current knowledge of neutrino oscillation parameters depends significantly on the experimental inputs that inform us of details of the production and interactions of neutrinos, which were presented by the NA61/SHINE hadron production experiment and cross section measurements from T2K and MINERvA, as well as a review of the status of our understanding of neutrino production at nuclear reactors. The session also included theoretical reviews of the current status of neutrino oscillations, and phenomenological studies on neutrino tomography and experimental studies to support nuclear matrix element calculations (NUMEN).
\end{abstract}

Neutrino Oscillation Workshop (NOW 2018)

9 - 16 September, 2018

Rosa Marina (Ostuni, Brindisi, Italy) 


\section{Introduction}

This is a particularly exciting time in the field of neutrino oscillation physics. Since the "third" type of oscillation was discovered at the beginning of the decade, we have finite measurements of all the mixing angles and the magnitudes of the mass-splittings within the framework of the standard Pontecorvo-Maki-Nakagawa-Sakata (PMNS) neutrino oscillation model. These measurements are continuously being improved through the existing experiments which are currently taking data, and experiments which have concluded their data-taking runs are now providing updated and final analyses-including some which continue to raise questions about the completeness of the PMNS description. Future experiments are being planned to help further this knowledge, and in Session I we also focus on those which aim to answer these questions as to whether the PMNS model is valid. Furthermore, the increasingly high precisions that experiments are achieving have brought to the fore the need for a much better understanding of, for example, the properties of neutrinos and other particles which influence the rates of neutrino production, and the nuclear physics which governs the interactions of neutrinos in our detectors. The information provided by these experiments and theoretical inputs are being combined in the form of "global fits" which point the way towards further progress in the near future.

In this Session of the NOW 2018 workshop, experts in these areas provided an experimental and theoretical survey of the present status of neutrino oscillations. Here we provide a brief summary of the presentations and discussions. Results, figures and references that are specific to the presentations can be found in the corresponding contributions in these proceedings.

\section{Current and Near-Future Oscillation Experiments}

The two currently-running long-baseline neutrino oscillation experiments opened the workshop by presenting their latest results.

The T2K experiment has analysed data that it collected between the years of 2010 and 2017, including with the beam running in antineutrino mode for almost half of the corresponding protonson-target (POTs) [1]. The beam is measured by three major detector suites: the on-axis "near" detector, INGRID, at $280 \mathrm{~m}$ from the pion-producing proton beam target; Super-Kamiokande, the "far" detector at a distance of $295 \mathrm{~km}$ and at a position that is about 2.5 degrees off the central neutrino beam axis, which lowers the peak beam energy and the level of background-producing higher-energy flux; and the ND280 detector, also at $280 \mathrm{~m}$ but with the same off-axis angle as Super-K. Much emphasis is placed on how the information from these detectors are combined, together with the knowledge of how neutrinos are produced in the beam as well as their interactions with nuclear matter-topics which are covered in more detail later in the Session $[18,16]$. The current data exclude charge-parity symmetry non-conservation in the neutrino sector at the $2 \sigma$ level, and a slight preference for the normal hierarchy for the ordering of the neutrino masses. Upgrades to all the major components of the experiment, including the neutrino-producing beam system, are being carried out to improve the experiment over the next few years.

The NOvA experiment presented results based on its dataset from 2014 till early 2018, which also includes close to half of the POTs in antineutrino mode [2]. It uses two functionally-identical detectors which are both placed at an off-axis angle of $14 \mathrm{mrad}$ ( 0.8 degrees), with the far detector 
at $810 \mathrm{~km}$ and the smaller near detector at $1 \mathrm{~km}$ from the proton beam target. Convolutional Neural Network techniques are applied for event selection at these detectors, without using any explicit event reconstruction information. The use of identical technologies at the detectors allow for firstorder cancellations of differences in beam flux aside from those caused by neutrino oscillations, but interaction models and simulations are used to understand further differences. Inputs from the MINERvA experiment [17] as well as NOvA near detector data are used to tune these. The results demonstrate the appearance of electron antineutrinos in a muon antineutrino beam at the $4 \sigma$ level, and the oscillation analysis favours the normal hierarchy at $1.8 \sigma$ and excludes, for the inverted hierarchy, a $\delta_{\mathrm{CP}}$ value of $\pi / 2$ at $3 \sigma$. No significant suppression of neutral current events which would be evidence for sterile neutrinos is seen, and several astrophysical measurements from NOvA are forthcoming. Data-taking is planned to continue until 2024, when a $3 \sigma$ measurement of the mass hierarchy is expected.

Atmospheric neutrinos remain a rich source of knowledge, more than two decades after the conclusive evidence of neutrino flavour change from Super-K [3]. Six very large dedicated experiments around the world are either underway or are being built, using underground tanks of water, the bottom of the sea, Antarctic ice, and magnetised iron as the interaction medium for neutrinos. Super-K is not only continuing to constrain the neutrino oscillation parameters, but now has sensitivity to the effect of the Earth's matter on atmospheric oscillations, and reports strong evidence of $v_{\tau}$ appearance. Significant improvements are on their way, including the addition of Gadolinium to the water, which will enable the detector to tag neutrons at $90 \%$ efficiency, which will allow the experiment to expand the physics that it is able to explore.

The IceCube experiment presented their latest atmospheric neutrino oscillation results, using three years of data [7]. The DeepCore infill array at the centre of IceCube enables the detection and reconstruction of neutrinos produced by the interaction of cosmic rays in the Earth's atmosphere at energies as low as $5 \mathrm{GeV}$. Muon neutrino disappearance has been measured over a range of baselines, probing the same $L / E$ as accelerator experiments but with higher-energy neutrinos. The absence of $v_{\tau}$ oscillations is excluded at $3.2 \sigma$, and new limits on sterile neutrino mixing in the muon and tau sectors were shown.

The ANTARES experiment, running since 2007, presented updated neutrino oscillation results with 10 years of data, which are consistent with the world best-fit $\theta_{23}$ and $\Delta m_{32}^{2}$ values [8]. The no-oscillation hypothesis is now excluded at $4.6 \sigma$. The $3+1$ sterile neutrino mode was tested and new constraints set on $\left|U_{\mu 4}^{2}\right|$ and $\left|U_{\tau 4}^{2}\right|$.

The ICARUS T600 detector was moved to Fermilab in 2017 after a major overhaul and will soon be exposed to the Booster Neutrino Beam, acting as the far detector, at a distance of $600 \mathrm{~m}$, to search for sterile neutrinos within the Short Baseline Neutrino (SBN) programme [12]. The upgrade of the detector at CERN included the installation of new cold vessels, renovated cryogenics and liquid argon (LAr) purification system, improved cathode planarity, upgraded light collection systems and new faster and higher performance TPC readout electronics. The rigging of the two modules and placement in the Fermilab far site building was completed in August 2018. The detector is expected to be commissioned and to start taking data in early 2019 .

MicroBooNE [11] is situated at $470 \mathrm{~m}$ along the same neutrino beam, just short of the baseline where the yet-to-be-understood low-energy excess was observed by MiniBooNE. It first aims to resolve this excess though the bubble chamber-like resolution offered by the LAr TPC, and will 
then contribute to the SBN sterile neutrino search effort. The experiment presented some preliminary results on neutrino cross-section measurements, as well as its current work focusing on the forthcoming low-energy excess search.

Reactor neutrino experiments still play an important role in the understanding of neutrino oscillations. A summary of the experimental results from Daya Bay, Double Chooz and RENO was presented [4], including the status of sterile neutrino searches and reactor neutrino spectrum. The most precise measurements of $\theta_{13}$ and $\Delta m_{e e}^{2}$ were reported together with an impressive $L / E$ dependence of the electron antineutrino oscillation probability. The best fit point for the Reactor Antineutrino Anomaly is disfavoured by the short-baseline reactor neutrino experiments such as PROSPECT, NEOS, DANNS and STEREO. The origin of the data/MC discrepancy on the reactor neutrino spectrum near $5 \mathrm{MeV}$ is still under investigation.

The most recent solar neutrino results from the Borexino experiment at the Gran Sasso underground laboratory were presented [14]. In particular, refined measurements of all neutrinos produced in the $p p$ fusion chain have been made, favouring a high-metallicity Sun. They reported a $5 \sigma$ evidence of pep solar neutrinos, including systematic uncertainties and a new limit on the CNO neutrino flux. New activities are in progress to understand the ${ }^{210} \mathrm{Po}$ evolution in the detector.

\section{Updated Results from Earlier Oscillation Experiments}

Double Chooz presented their $v_{e}$ disappearance results with two detectors at distances of 400 and $1050 \mathrm{~m}$ from the Chooz nuclear reactors, and report a $2 \sigma$ tension between their current $\theta_{13}$ result and that from Daya Bay [13]. Near detector results add to the mounting evidence for a deficit of $\bar{v}_{e}$ production relative to expectations.

MiniBooNE presented a recent update of their measurement of electron-like events appearing in a $v_{\mu}$ beam which had originally resulted in the low-energy excess at neutrino energies of below $600 \mathrm{MeV}$ [10]. The new data make use of twice the neutrino beam exposure, and the new analysis, which preserves the selection cuts used, results in the significance of the combined excess for neutrino and antineutrino modes being raised to $4.7 \sigma$.

The OPERA experiment measured in 2015 the appearance of $v_{\tau}$ events in the CNGS $v_{\mu}$ beam with 5.1 $\sigma$ significance [9]. An updated analysis was presented using a looser event selection, doubling the $v_{\tau}$ sample with negligible additional background. A value of $\left|\Delta m_{32}^{2}\right|=\left(2.7_{-0.6}^{+0.7}\right) \times$ $10^{-3} \mathrm{eV}^{2}$ was measured for the first time in appearance mode, assuming maximal mixing. Updated results on $v_{\mu} \rightarrow v_{e}$ oscillation searches, $v_{\mu}$ disappearance and exclusion regions on $3+1$ sterile neutrino oscillation parameters were presented.

\section{Theoretical and Experimental Considerations for Measurements of Neutrino Properties}

Many, if not all, of the experiments above depend on significant inputs from other experiments and theoretical work to be able to make their measurements, and several of these were also presented in Session I.

The prediction of the neutrino flux and energy spectrum emitted by nuclear reactors continues to present a challenge, and has evolved quite significantly over the years. A comprehensive survey 
was given [5], which focused on topics including: a-priori calculations based on $\beta$-decay and fission theory, considering much recent work on detailed corrections to these such as the impact of forbidden decays; measured fission yield data-where significant inconsistencies have been identified in the past few years; and the results from short-baseline reactor neutrino experiments. The puzzle posed by the distortion at $5 \mathrm{MeV}$ which is seen in experiments is being explored, with the conclusion that more measurements at different reactors will be needed to reach the precision that is needed.

The information provided by the T2K near detector, ND280, is crucial in allowing the experiment to make oscillation measurements at the level that it is able to. A selection of results from the almost 40 different analysis channels that are being studied at ND280, on both water and plastic targets, was presented [16]. These individual measurements are compared with a variety of neutrino-nucleus interaction models to help achieve a consistent understanding, and it was demonstrated that more data and analysis are needed to make further progress.

MINERvA [17] is a dedicated experiment for investigating neutrino interactions, positioned near the source of the beam that also serves NOvA. It operates at the several-GeV range in neutrino energy, complementing the $1 \mathrm{GeV}$ events studied at the T2K near detector, and in addition to the interaction cross-section information it provides, MINERvA also helps the NOvA experiment by reducing the uncertainties on the neutrino beam flux estimation.

NA61/SHINE at CERN is a dedicated experiment for making measurements to help us understand the production rates and kinematics of the hadrons which then decay to produce the neutrinos used by long-baseline experiments [18]. These are produced by proton beams of many GeV being sent into targets-normally cylindrical rods made of materials such as graphite and aluminium and heavier metals. There are significant uncertainties in the QCD-dominated predictions for hadron production, and NA61 has made measurements with full replica targets and thin slices of target to help understand the T2K and NOvA beams. A new project at Fermilab called EMPHATIC, which focuses on low-momentum studies which complement NA61, was also introduced.

New results from the NUMEN experiment were shown [19]. NUMEN proposes an innovative technique to access the nuclear matrix elements entering the expression of the lifetime of the double beta decay by cross section measurements of heavy-ion induced Double Charge Exchange (DCE) reactions. First experimental results obtained at the INFN-LNS laboratory for the ${ }^{40} \mathrm{Ca}\left({ }^{18} \mathrm{O},{ }^{18} \mathrm{Ne}\right){ }^{40} \mathrm{Ar}$ reaction at $270 \mathrm{MeV}$ gave encouraging indications on the capabilities of the technique. The two major components in this project are the K800 Superconducting Cyclotron and MAGNEX spectrometer. An upgrade of the LNS accelerator and beam lines, together with the experimental set-up, is needed to achieved the resolution requirements. Recent results with ${ }^{20} \mathrm{Ne}$ beams were shown together with plans for ${ }^{18} \mathrm{O}$ beams.

J. Salvado presented a study of Earth tomography with neutrinos using the publicly available one-year through-going muon sample of the atmospheric neutrino data from IceCube [15]. These results confirm that current neutrino detectors are able to determine the mass of the Earth, its moment of inertia, the mass of the Earth's core and establish that the core is denser than the mantle, using weak interactions only, in a way that is completely independent of gravitational measurements. The results are dominated by statistical uncertainties and they can be improved using more data from current and future neutrino detectors.

Finally, I. Martínez-Soler showed the status of the combined fit to global neutrino oscillation 
data available as of Summer 2018 in the scenario of three-neutrino oscillations [6]. This includes data from all solar neutrino experiments, reactor data from KamLAND as well as from the shortbaseline reactor experiments Daya Bay, RENO and Double Chooz, atmospheric data from IceCube/DeepCore, together with long-baseline accelerator data from MINOS, T2K and NOvA. The combination of all data sets allows for a very accurate determination of the neutrino oscillation parameters $(8-24 \%)$. However, the main unknowns in the three-neutrino oscillation scenario remain unresolved. There is a slight preference for $\theta_{23}$ in the second octant. The best fit of the analysis is for the normal mass ordering at more than $3 \sigma$ and for the complex phase $\delta_{C P}=234^{\circ}$ with $\mathrm{CP}$ conservation being allowed at around $2 \sigma$.

\section{Conclusion}

The contributions to Session I demonstrated that the study of neutrino oscillations is now an area where numerous experiments are allowing precision measurements of increasingly high precision to be made in many complementary ways, pinning down the PMNS model parameters one by one, whilst still keeping open many questions that will ensure it remains an exciting field for many years to come.

\section{References}

[1] Eric Zimmerman, "T2K latest results”, talk given at NOW 2018; these proceedings (2018) [as with all references below]

[2] Matthew Strait, "NOVA latest results"

[3] Cristovao Vilela, "Atmospheric neutrino oscillations"

[4] Zhe Wang, "Reactor neutrino oscillations"

[5] Patrick Huber, "Reactor spectral shape challenges"

[6] Ivan Martinez-Soler, "Global status of three-neutrino mixing"

[7] Andrii Terliuk, "Atmospheric oscillations with IceCube"

[8] Juergen Brunner, "ANTARES atmospheric oscillations"

[9] Gabriele Sirri, "Final results from OPERA"

[10] Michael Shaevitz, "Updated electron- $v$ appearance results from MiniBooNE"

[11] Matthew Toups, "MicroBooNE"

[12] Gianluca Raselli, "Sterile searches with the ICARUS detector"

[13] Thiago Sogo Bezerra, "Double Chooz results at the double detector phase"

[14] Gemma Testera, "Results from Borexino on solar and geo-neutrinos"

[15] Jordi Salvado, "Neutrino tomography of the earth"

[16] Margherita Buizza Avanzini, "T2K -nucleus cross section results”

[17] Jorge Chaves, "MINERvA results"

[18] Matej Pavin, "NA61/SHINE measurements for physics"

[19] Clementina Agodi, "New results from the NUMEN project" 